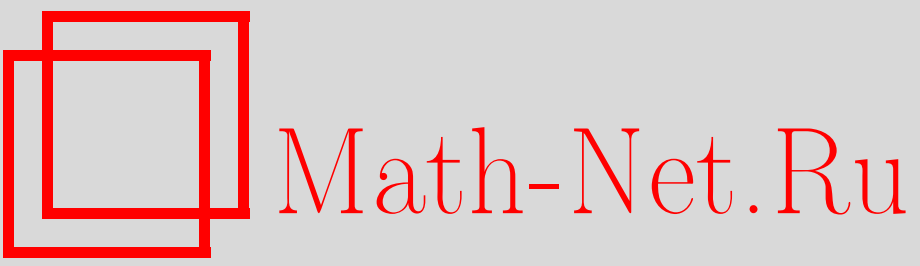

Ю. Г. Строганов, Квазисвободные состояния в некоторых одномерных квантовых спиновых моделях, ТМФ, 2004, том 139, номер 1, 112-128

DOI: https://doi.org/10.4213/tmf46

Использование Общероссийского математического портала Math-Net.Ru подразумевает, что вы прочитали и согласны с пользовательским соглашением

http://www.mathnet.ru/rus/agreement

Параметры загрузки:

IP : 54.157 .27 .8

26 апреля 2023 г., $12: 16: 23$ 


\title{
КВАЗИСВОБОДНЫЕ СОСТОЯНИЯ В НЕКОТОРЫХ ОДНОМЕРНЫХ КВАНТОВЫХ СПИНОВЫХ МОДЕЛЯХ
}

\begin{abstract}
С использованием численных методов исследована $S U_{q}(N)$-спиновая цепочка Перка-Шульц со специальным значением квантового параметра $q=-e^{i \pi / N}$. Обнаружены простые закономерности в значительной части спектра гамильтониана, к которой, в частности, принадлежит энергия основного состояния и ближайшие возбуждения. Полученные феноменологические формулы напоминают формулы для спектра модели свободных фермионов. Сформулировано несколько гипотез, часть из которых удается обосновать, строя точные решения системы уравнений анзаца Бете для цепочек конечной длины. Получено два множества решений для этих уравнений. Первое соответствует специальному значению квантового параметра $q$ и описывает, в частности, основное состояние модели, которое носит антиферромагнитный характер. Второе описывает часть спектра, принадлежащую секторам, где числа $n_{i}$ частиц разного типа $(i=0,1, \ldots, N-1)$ меньше или равны единице для всех типов, кроме одного. Для этого множества получен простой спектр при произвольном значении параметра $q$. Предполагается, что данный спектр и найденные в замкнутом виде решения уравнений анзаца Бете тесно связаны с существованием специального собственного состояния для трансфер-матрицы вспомогательной неоднородной $S U_{q}(N-1)$-вершинной модели, которая фигурирует при построении системы уравнений анзаца Бете, имеющей структуру "матрешки". Приведены косвенные аргументы в пользу этой гипотезы, основанные на комбинаторных свойствах волновой функции рассматриваемого состояния.
\end{abstract}

Ключевые слова: модель Перка-Шульц, конечные спиновые цепочки, точные решения уравнений анзаца Бете.

\section{1. ВВЕДЕНИЕ}

Со времени пионерской работы Бете [1] анзац Бете и его различные обобшения оказались эффективным орудием при описании собственных векторов различных квантовых цепочек и трансфер-матриц двумерных решетчатых статистических моделей (см, например, [2]). Модели с волновой функцией, описываемой анзацем Бете, называются интегрируемыми. Согласно этому анзацу, волновые функции являются суммой плоских волн, чьи волновые числа определяются с помощью системы нелинейных рациональных, тригонометрических или эллиптических уравнений. В термодинамическом пре-

* Институт физики высоких энергий, Протвино, Московская обл., Россия. E-mail: stroganov@mx.ihep.su 
деле удается, как правило, свести эти уравнения к решаемым системам интегральных уравнений.

Можно, однако, встать на ту точку зрения, что бете- (янг-бакстер-) интегрируемость формулируется независимо от размера системы, и что точные результаты для конечных систем или точные решения соответствуюших уравнений Бете могли бы служить важным шагом по пути более полного математического и физического понимания этих моделей.

Из-за сложности уравнений лишь в двух специальных случаях получены аналитические решения для конечных систем произвольного размера. Первый случай - это знаменитая $X Y$-спиновая цепочка, сводяшаяся к свободным фермионам (Либ, Шульц и Маттис) и некоторые родственные модели, для которых система уравнений Бете расцепляется на простые отдельные уравнения или небольшие подсистемы уравнений. Второй случай начал изучаться сравнительно недавно. Он относится к $X X Z$-спиновой цепочке со специальным параметром анизотропии $\Delta=-1 / 2\left(q=e^{2 i \pi / 3}\right)[3]-[5]$. В этом случае решение уравнений Бете для основного состояния удалось построить точно [5], используя $T-Q$ уравнения Бакстера [6]. Был найден $Q(u)$-многочлен, чьи нули дают волновые числа Бете. Оказалось, что этот многочлен тесно связан с интересной комбинаторной структурой - матрицами чередующихся знаков. В самое последнее время эта модель и родственные ей модели привлекли большое внимание [7]. Однако, хотя и удалось установить некоторые точные свойства соответствуюших волновых функций, полное, замкнутое вычисление их амплитуд все еще отсутствует ${ }^{1}$.

В данной работе кратко изложены результаты работ [9], вьполненных во время пребывания автора в Бразилии. Основным результатом этих работ является изучение $S U_{q}(N)$-обобщений описанных выше двух специальных случаев точного решения уравнений Бете для конечных систем. В разделе 2 введены два варианта $S U_{q}(N)$-модели Перка-Шульц, которые и будут рассматриваться в дальнейшем. Первый вариант соответствует периодическим граничным условиям, второй - открытым. В разделе 3 формулируется несколько гипотез, основанных на результатах численной диагонализации гамильтониана модели. Мы ограничиваемся случаем $N=3$ и периодическими граничными условиями. Этот случай был исторически самым первым, но нам не удалось его обобщить на значения $N>3$. Что касается случая открытых граничных условий, то полученные численные результаты, которые, по всей вероятности, обобщаются на произвольные значения $N$, изложены в разделе 4 . В разделе 5 выписаны соответствуюшие уравнения Бете. Кроме того, нами сужен класс рассматриваемых решений с помощью наложения дополнительных "условий свободных фермионов". Точные решения уравнений Бете для простейшего случая $S U_{q}(3)$-спиновой цепочки с периодическими граничными условиями приведены в разделе 6 . В разделе 7 содержится аналогичное, хотя и более сложное, рассмотрение случая $S U_{q}(3)$-спиновой цепочки с открытой границей. Раздел 8 содержит рассмотрение обшей проблемы построения свободных фермионных состояний. В разделе 9 приводятся результаты изучения комбинаторных свойств функций основного состояния $S U_{q}(N-1)$-модели с параметром $q=-e^{i \pi / N}$.

\footnotetext{
${ }^{1)} \mathrm{Cм.} \mathrm{также} \mathrm{работы} \mathrm{[4],} \mathrm{[8]} \mathrm{для} \mathrm{обобщения} \mathrm{на} X Y Z$-спиновую цепочку нечетной длины.
} 


\section{2. $S U_{q}(N)$-МОДЕЛЬ ПЕРКА-ШУЛЬЦ}

$S U_{q}(N)$-модель Перка-Шульц [10] является анизотропным обобщением $S U(N)$-модели Сазерленда [11] с гамильтонианом

$$
H_{q}^{p}=\sum_{j=1}^{L-1} H_{j, j+1}+p H_{L, 1}, \quad p=0,1,
$$

где

$$
H_{i, j}=-\sum_{a=0}^{N-1} \sum_{b=a+1}^{N-1}\left(E_{i}^{a b} E_{j}^{b a}+E_{i}^{b a} E_{j}^{a b}-q E_{i}^{a a} E_{j}^{b b}-q^{-1} E_{i}^{b b} E_{j}^{a a}\right),
$$

определенным на цепочке длины $L . \quad(N \times N)$-матрицы $E^{a b}$ имеют матричные элементы $\left(E^{a b}\right)_{c d}=\delta_{c}^{a} \delta_{d}^{b}$, а квантовый параметр $q=e^{i \eta}$ определяет анизотропию модели. Полагая $p=1$ или $p=0$ в формуле (1), мы получим случаи с периодическими или со свободными граничными условиями, соответственно. Гамильтониан описывает динамику системы, содержашей $N$ сортов частиц, причем в каждой ячейке цепочки располагается в точности одна частища. Число частиц каждого сорта сохраняется и, следовательно, пространство состояний распадается на несвязанные сектора, которые мы будем обозначать $\left(n_{0}, n_{1}, \ldots, n_{N-1}\right)$, где $n_{i} \quad\left(0 \leqslant n_{i} \leqslant L\right)$ - обшее число частиц типа $i \quad(i=0,1, \ldots, N-1)$. Гамильтониан обладает $S_{N}$-симметрией по отношению к перестановкам типов частищ, поэтому все собственные значения гамильтониана могут быть получены при изучении секторов $\left(n_{0}, n_{1}, \ldots, n_{N-1}\right)$, где $0 \leqslant n_{0} \leqslant n_{1} \leqslant \cdots \leqslant n_{N-1} \leqslant L$ и $n_{0}+n_{1}+\cdots+n_{N-1}=L$. Более того, в специальном случае свободных граничных условий $(p=0)$ квантовая цепочка (1) обладает также добавочной квантовой $S U_{q}(N)$-симметрией. В результате все энергии сектора $\left(n_{0}^{\prime}, n_{1}^{\prime}, \ldots, n_{N-1}^{\prime}\right)$ с $0 \leqslant n_{0}^{\prime} \leqslant n_{1}^{\prime} \leqslant \cdots \leqslant n_{N-1}^{\prime} \leqslant L$ встречаются также в секторах $\left(n_{0}, n_{1}, \ldots, n_{N-1}\right)$ с $0 \leqslant n_{0} \leqslant n_{1} \leqslant \cdots \leqslant n_{N-1} \leqslant L$, если $n_{0}^{\prime} \leqslant n_{0}$, $n_{0}^{\prime}+n_{1}^{\prime} \leqslant n_{0}+n_{1}, \ldots, n_{0}^{\prime}+n_{1}^{\prime}+\cdots+n_{N-2}^{\prime} \leqslant n_{0}+n_{1}+\cdots+n_{N-2}$.

\section{3. СВОБОДНО-ФЕРМИОННЫЙ СПЕКТР ДЛЯ ПЕРИОДИЧЕСКОЙ $S U_{q}(3)$-КВАНТОВОЙ ЦЕПОЧКИ С $q=e^{2 i \pi / 3}$. ЧИСЛЕННЫЕ РЕЗУЛЬТАТЫ}

Сформулируем несколько гипотез, полученных в результате численной диагонализации гамильтониана (1) для $N=3, p=1$ при $q=e^{2 i \pi / 3}$ с помощью компьютера. Часть этих гипотез будет обоснована в следуюшем разделе путем прямого решения соответствуюших уравнений Бете.

ГипотеЗА 1. Гамильтониан (1) для цепочки длинъ L при $q=e^{2 i \pi / 3}$ имеет собственные векторы с әнергией и импульсом

$$
\begin{aligned}
E_{I} & =-\sum_{j \in I}\left(1+2 \cos \frac{2 \pi j}{L}\right), \\
P_{I} & =\frac{2 \pi}{L} \sum_{j \in I} j
\end{aligned}
$$


где $I$ является $\mathcal{I}$-элементным подмножеством мнодества $\{1,2, \ldots, L\}$. Число $\mathcal{I}$ должсно быть нечетным $(\mathcal{I}=2 k+1)$, и сектор появления указанных уровней определяется параметром $k: S_{k} \equiv(k, k+1, L-2 k-1), \quad 0 \leqslant k \leqslant(L-1) / 2$.

Наименшшее значение энергии среди даваемых формулой (2) значений получается для подмножества $I_{0}^{(k)}=\{1,2, \ldots, k\} \cup\{L-k, \ldots, L\}$, так как в этом случае все слагаемые, входящие в сумму в формуле (2), имеют наименьшие возможные значения. Соответствуюшее состояние имеет импульс, равньй нулю, а энергия дается формулой

$$
E_{0}^{(k)}=-\sum_{j \in I_{0}^{(k)}}\left(1+2 \cos \frac{2 \pi j}{L}\right)=-2 k-1-2 \frac{\sin \frac{\pi(2 k+1)}{L}}{\sin \frac{\pi}{L}}
$$

ГИПотЕЗА 2. Для длинь иепочки $L=3 n+l \quad(l=1,2,3)$ u при $q=e^{2 i \pi / 3}$ cобственная әнергия $E_{0}^{(k)}$ является наименьшей в секторе $S_{k}$, кроме того, при $l \neq 3$ $(L \neq 3 n)$ формула

$$
E_{0}^{(n)}=-2 n-1-2 \frac{\sin \frac{\pi(2 n+1)}{L}}{\sin \frac{\pi}{L}}
$$

определяет энергию основного состояния модели.

Пусть теперь длина цепочки $L$ нечетна: $L=2 m+1$. Тогда, полагая $k=m$ в гипотезе 1 и используя единственно возможное в этом случае подмножество $I=\{1,2, \ldots L\}$, мы получаем собственный вектор с энергией $E=-L$ и с импульсом $P=0$. Этот вектор принадлежит сектору $S_{m}=(m, m+1,0)$, т.е., по сути дела, он принадлежит $S U_{q}(2)$ $(X X Z-)$ модели.

ГиПОтеЗА 3. Для $S U_{q}(2)-$ цепочки нечетной длины $L=2 m+1$ и при $q=e^{2 i \pi / 3}$ основное состояние принадлехит сектору $(m, m+1)$ и имеет энергию $E=-L$.

\section{4. СВОБОДНО-ФЕРМИОННЫЙ СПЕКТР ДЛЯ ОТКРЫТОЙ $S U_{q}(N)$-КВАНТОВОЙ ЦЕПОЧКИ С $q=-e^{i \pi / N}$. ЧИСЛЕННЫЕ РЕЗУЛЬТАТЫ}

Введем понятие специальных секторов. Специальными секторами будем называть пронумерованные секторы вида

$$
S_{k}=\left(\left[\frac{k}{N-1}\right],\left[\frac{k+1}{N-1}\right], \ldots,\left[\frac{k+N-2}{N-1}\right], L-k\right), \quad k=0,1, \ldots, L,
$$

где $[x]$ обозначает целую часть числа $x$. Рассмотрим, например, $N=4$ и $L=7$. Таблица специальных секторов выглядит следующим образом:

$$
\begin{aligned}
& S_{0}=(0,0,0,7), \quad S_{1}=(0,0,1,6), \quad S_{2}=(0,1,1,5), \quad S_{3}=(1,1,1,4), \\
& S_{4}=(1,1,2,3), \quad S_{5}=(1,2,2,2), \quad S_{6}=(2,2,2,1), \quad S_{7}=(2,2,3,0) \text {. }
\end{aligned}
$$


Благодаря квантовой симметрии, мы имеем упорядочение

$$
S_{0} \subset S_{1} \subset S_{2} \subset S_{3} \subset S_{4} \subset S_{5} \equiv S_{6} \supset S_{7}
$$

Это означает, что все собственные значения гамильтониана (1), найденные, например, в секторе $S_{3}$, найдутся также в секторах $S_{4}, S_{5}$ и $S_{6}$. С другой стороны, все собственные значения, принадлежашие сектору $S_{7}$, найдутся в секторах $S_{6}$ и $S_{5}$. В нашем примере секторы $S_{5}$ и $S_{6}$, очевидно, эквивалентны. Назовем секторы $S_{0}, S_{1}, S_{2}, S_{3}, S_{4}, S_{5}$ левыми секторами, а $S_{6}, S_{7}$ - правыми. Эти определения легко обобшаются на произвольное $L=N n+r$, где $n$ и $r-$ натуральные числа и $r<N$. В обшем случае мы получаем $L-n$ левых секторов и $n+1$ правых. Значения, полученные при численной диагонализации, позволяют сформулировать следуюшие гипотезы.

ГипотеЗА 4. Для $L=N n+r$ гамильтониан (1) с $p=0 u q=e^{i \pi / N}$ имеет собственные значения, описываемие формулой

$$
E_{I}=-2 \sum_{j \in I}\left(\cos \frac{\pi}{N}+\cos \frac{j \pi}{L}\right)
$$

где I - произвольное подмножество множества $\{1,2, \ldots, L-1\}$. Пусть $k$ - число әлементов подмножества I и пусть $S_{k}$ - левый сектор. Тогда мъ найдем собственное значение (9) в секторах $S_{k}, S_{k+1}, \ldots, S_{L-n}$. Наоборот, если $S_{k}$ является правым сектором, то мы найдем это собственное значение в секторах $S_{L-n-1}, S_{L-n}, \ldots, S_{k+1}$

При $L=N n$ имеем другую картину. Для этого случая наряду с левыми и правыми секторами имеется еще центральный сектор $(n, n, n, n)$.

ГИпотезА $4^{\prime}$. Для $L=N n$ мь можем использовать гипотезу 4 , рассматривая центральный сектор или как левыц й, или как правый.

Это возможно, потому что спектры в секторах $(n, n, n, \ldots, n)$ и $(n-1, n, n, \ldots, n+1)$ совпадают с точностью до кратности.

Рассмотрим теперь специальные подмножества $I_{k}=1,2,3, \ldots, k, k=0,1, \ldots, L-1$.

Согласно гипотезам 4 и $4^{\prime}$ гамильтониан (1) имеет собственные значения

$$
E^{(k)}=-2 \sum_{j=1}^{k}\left(\cos \frac{\pi}{N}+\cos \frac{j \pi}{L}\right)=1-2 k \cos \frac{\pi}{N}-\frac{\sin \frac{\pi(2 k+1)}{2 L}}{\sin \frac{\pi}{2 L}} .
$$

ГиПотеЗА 5. Формула (10) дает значение для минимального собственного значения гамильтониана (1) при $q=-e^{i \pi / N}$ в специальных секторах (6). А именно, для левых секторов выполняется равенство $E_{\min }\left(S_{k}\right)=E^{(k)}$, равенство $E_{\min }\left(S_{k}\right)=E^{(k-1)}$ справедливо для правых секторов. 
ГипотеЗА $5^{\prime}$. Формула (10) для $k=L-n-1$ дает әнергию основного состояния гамильтониана (1),

$$
E_{0}=1+2(1-L+n) \cos \frac{\pi}{N}-\frac{\sin \frac{\pi(2 n+1)}{2 L}}{\sin \frac{\pi}{2 L}} .
$$

Напомним, что $n=[L / N]$.

Последним из специальных секторов является сектор

$$
S_{L}=\left(\left[\frac{L}{N-1}\right],\left[\frac{L+1}{N-1}\right], \ldots,\left[\frac{L+N-2}{N-1}\right], 0\right), \quad k=0,1, \ldots, L .
$$

В нем мы имеем лишь $N-1$ сортов частиш и модель, по сути, сводится к $S U_{q}(N-1)$ инвариантной модели. Этот сектор является правым, и на основании гипотез 4 и $4^{\prime}$ мы можем сформулировать следуюшую гипотезу.

ГипотезА 6. Формула (10) для $k=L-1$ дает минимальное собственное значение (әнергию основного состояния) $S U_{q}(N-1)$-гамильтониана для $q=-e^{i \pi / N}$,

$$
E_{0}=-2(L-1) \cos \frac{\pi}{N}
$$

Например, для $N=3$ получаем, что $X X Z$-модель с $\Delta=-1 / 2$ имеет энергию основного состояния $E_{0}=1-L$.

\section{5. УРАВНЕНИЯ БЕТЕ}

Вариант уравнений Бете для $S U_{q}(N)$-модели Перка-Шульц имеет вид (см., например, [12]-[14])

$$
\prod_{j=1, j \neq i}^{p_{k}} F\left(u_{i}^{(k)}, u_{j}^{(k)}\right)=\prod_{j=1}^{p_{k-1}} f\left(u_{i}^{(k)}, u_{j}^{(k-1)}\right) \prod_{j=1}^{p_{k+1}} f\left(u_{i}^{(k)}, u_{j}^{(k+1)}\right),
$$

где $k=0,1, \ldots, N-2, i=1,2, \ldots, p_{k}$. Целочисленные параметры $p_{k}$ зависят от секто$\mathrm{pa}$,

$$
p_{k}=\sum_{i=0}^{k} n_{i}, \quad k=0,1, \ldots, N-2 .
$$

Функции $F(x, y)$ и $f(x, y)$ определены формулами

$$
F(x, y)=\frac{\sin (x-y-\eta)}{\sin (x-y+\eta)}, \quad f(x, y)=\frac{\sin \left(x-y-\frac{\eta}{2}\right)}{\sin \left(x-y+\frac{\eta}{2}\right)}
$$

в периодическом случае и

$$
F(x, y)=\frac{\cos (2 y)-\cos (2 x-2 \eta)}{\cos (2 y)-\cos (2 x+2 \eta)}, \quad f(x, y)=\frac{\cos (2 y)-\cos (2 x-\eta)}{\cos (2 y)-\cos (2 x+\eta)}
$$


для свободных граничных условий. В уравнениях (14) мы имеем дело с переменными разного порядка. Число переменных $u_{i}^{(k)}$ порядка $k$ равно $p_{k}$. Вся система уравнений Бете состоит из подсистем порядка $k(k=0,1, \ldots, N-2)$, содержащих в точности $p_{k}$ уравнений. В подсистеме наивысшего порядка $N-2$ формально появляются $L$ переменных порядка $N-1$. В нашем случае их следует положить равными нулю.

Собственные энергии гамильтониана (1) в секторе $\left(n_{0}, n_{1}, \ldots, n_{N-1}\right)$ даются уравнением

$$
E=-\sum_{j=1}^{p_{N-2}}\left(-q-\frac{1}{q}+\frac{\sin \left(u_{j}-\frac{\eta}{2}\right)}{\sin \left(u_{j}+\frac{\eta}{2}\right)}+\frac{\sin \left(u_{j}+\frac{\eta}{2}\right)}{\sin \left(u_{j}-\frac{\eta}{2}\right)}\right),
$$

где вместо $u_{j}^{(N-2)}$ мы пишем $u_{j}, j=1,2, \ldots, p_{N-2}$.

Все специальные решения уравнений Бете (14), описываемые в этой статье, удовлетворяют дополнительным "условиям свободных фермионов"

$$
f^{L}\left(u_{i}, 0\right)=1, \quad i=1, \ldots, p_{N-2},
$$

и формула (18) для энергий гамильтониана (1) может быть записана в виде

$$
E=-2 \sum_{j=1}^{p_{N}-2}\left(-\cos \eta+\cos \frac{\pi k_{j}}{L}\right), \quad 1 \leqslant k_{j} \leqslant L-1
$$

для свободного случая. Для случая периодических граничных условий решения удалось найти только для $S U_{q}(3)$-модели с параметром $\eta=2 \pi / 3$. Формула (18) приобретает вид

$$
E=-\sum_{j=1}^{p_{1}}\left(1+2 \cos \frac{2 \pi k_{j}}{L}\right), \quad 1 \leqslant k_{j} \leqslant L .
$$

Общее число уравнений для “свободно-фермионного” случая превышает число переменных на $p_{N-2}$. На первый взгляд кажется, что удовлетворить всей системе уравнений невозможно. Оказывается, однако, что рассматриваемые нами уравнения Бете обладают некоторой скрытой симметрией. В следуюшем разделе мы рассмотрим простейший случай $S U_{q}(3)$-модели с периодическими граничными условиями.

\section{6. РЕШЕНИЯ УРАВНЕНИЙ БЕТЕ ДЛЯ $S U_{q}(3)-$ МОДЕЛИ \\ С ПЕРИОДИЧЕСКИМИ ГРАНИЧНЫМИ УСЛОВИЯМИ}

Уравнения Бете $(14)$ для $S U_{q}(3)$-модели Перка-Шульц, дополненные условием свободных фермионов при $q=e^{2 i \pi / 3}$ могут быть записаны следующим образом:

$$
\begin{aligned}
\prod_{j=1, j \neq i}^{p_{0}} f\left(v_{i}, v_{j}\right) \prod_{j=1}^{p_{1}} f\left(v_{i}, u_{j}\right) & =1, \quad i=1,2, \ldots, p_{0} \\
\prod_{j=1, j \neq i}^{p_{1}} f\left(u_{i}, u_{j}\right) \prod_{j=1}^{p_{0}} f\left(u_{i}, v_{j}\right) & =1, \quad i=1,2, \ldots, p_{1} \\
f^{L}\left(u_{i}, 0\right) & =1, \quad i=1,2, \ldots, p_{1}
\end{aligned}
$$


где $p_{0}=n_{0}, p_{1}=n_{0}+n_{1}$. Мы использовали тот факт, что при $q=e^{2 i \pi / 3}$, т.е. для $\eta=2 \pi / 3$ справедливо равенство

$$
F(x, y)=\frac{1}{f(x, y)}
$$

Прежде всего рассмотрим для примера сектор $(1,2, L-3)$. Тогда $p_{0}=n_{0}=1$ и $p_{1}=n_{0}+n_{1}=3$. Для этого простого случая первая подсистема (22) состоит из одного уравнения

$$
f\left(v, u_{1}\right) f\left(v, u_{2}\right) f\left(v, u_{3}\right)=1 .
$$

Подставив формулу для функции $f(16)$ в последнее уравнение, получим после простых преобразований уравнение

$$
\cos \left(v+u_{1}-u_{2}-u_{3}\right)+\cos \left(v-u_{1}+u_{2}+u_{3}\right)+\cos \left(v-u_{1}-u_{2}+u_{3}\right)=0 .
$$

Ясно, что оно имеет $S_{3}$-симметрию при перестановках переменных $u_{1}, u_{2}$ и $u_{3}$. Удивительно, что это уравнение симметрично по всем входящим в него четырем переменным!

Три уравнения второй подсистемы (23) получаются из уравнения (22) перестановкой $v_{i} \leftrightarrow u_{i}$, поэтому они также сводятся к симметричному уравнению (27). Фиксируя переменные $u_{i}, i=1,2,3$, так, чтобы они удовлетворяли условиям свободных фермионов $(24)$ и находя $v$ из уравнения (27), получаем решение для всей системы $(22)-(24)$ в секторе $(1,2, L-3)$.

Покажем сейчас, что такое рассмотрение обобщается на любой сектор вида $(k, k+1$, $L-2 k-1)$, обосновывая тем самым гипотезу 1 . Объединим две системы переменных

$$
x_{i}=v_{i}, \quad i=1,2, \ldots, p_{0} ; \quad x_{i+p_{0}}=u_{i}, \quad i=1,2, \ldots, p_{1} .
$$

Система уравнений (22)-(24) принимает вид

$$
\begin{array}{ll}
r\left(x_{i}\right)=f\left(x_{i}, x_{i}\right), & i=1,2, \ldots, p_{0}+p_{1}, \\
f^{L}\left(x_{i}, 0\right)=1, & i=p_{0}, p_{0}+1, \ldots, p_{0}+p_{1},
\end{array}
$$

где $r(x) \equiv \prod_{j=1}^{p_{0}+p_{1}} f\left(x, x_{j}\right)$. Подсистема (29) обладает $S_{p_{0}+p_{1}}$-перестановочной симметрией. Покажем, что в ней довольно много зависимых уравнений и что при $p_{0}=k$ и $p_{1}=2 k+1$ можно также удовлетворить условиям свободных фермионов. Подставим определение (16) в подсистему (29). По определению

$$
r(x)=\prod_{j=1}^{p_{0}+p_{1}} \frac{\sin \left(x-x_{j}-\frac{\pi}{3}\right)}{\sin \left(x-x_{j}+\frac{\pi}{3}\right)}=\prod_{j=1}^{p_{0}+p_{1}} \frac{b-q b_{j}}{q b-b_{j}}
$$

где

$$
b=e^{2 i x}, \quad b_{j}=e^{2 i x_{j}}, \quad q=e^{2 i \pi / 3} .
$$


Подсистема (29) преврашается в систему алгебраических уравнений

$$
\prod_{j=1}^{p_{0}+p_{1}}\left(b_{i}-q b_{j}\right)+\prod_{j=1}^{p_{0}+p_{1}}\left(q b_{i}-b_{j}\right)=0, \quad i=1,2, \ldots, p_{0}+p_{1} .
$$

Используя стандартные симметричные функции

$$
\begin{aligned}
& S_{0}=1 \text {, } \\
& S_{1}=b_{1}+b_{2}+\cdots+b_{p_{0}+p_{1}}, \\
& S_{2}=b_{1} b_{2}+b_{1} b_{3}+\cdots+b_{p_{0}+p_{1}-1} b_{p_{0}+p_{1}}, \\
& S_{m}=\sum_{1 \leqslant i_{1}<i_{2}<\cdots<i_{m} \leqslant p_{0}+p_{1}} b_{i_{1}} b_{i_{2}} \ldots b_{i_{m}}, \\
& S_{p_{0}+p_{1}}=b_{1} b_{2} \ldots b_{p_{0}+p_{1}},
\end{aligned}
$$

можно переписать уравнение (33) в виде

$$
\sum_{m=0}^{p_{0}+p_{1}}(-1)^{m} S_{m} b_{i}^{p_{0}+p_{1}-m}\left(q^{m}+q^{p_{0}+p_{1}-m}\right)=0 .
$$

С другой стороны, справедливо равенство

$$
q^{-p_{0}-p_{1}} \prod_{j=1}^{p_{0}+p_{1}}\left(b_{i}-b_{j}\right) \equiv \sum_{m=0}^{p_{0}+p_{1}}(-1)^{m} S_{m} b_{i}^{p_{0}+p_{1}-m} q^{-p_{0}-p_{1}}=0 .
$$

Складывая равенство (36) с уравнением (35), получаем

$$
\sum_{m=0}^{p_{0}+p_{1}}(-1)^{m} S_{m} b_{i}^{p_{0}+p_{1}-m}\left(q^{m}+q^{p_{0}+p_{1}-m}+q^{-p_{0}-p_{1}}\right)=0 .
$$

Для $q=e^{2 i \pi / 3}$ имеем соотношения

$$
\begin{array}{llll}
q^{m}+q^{p_{0}+p_{1}-m}+q^{-p_{0}-p_{1}}=3 q^{-p_{0}-p_{1}} & \text { при } & p_{0}+p_{1}+m=3 n, \\
q^{m}+q^{p_{0}+p_{1}-m}+q^{-p_{0}-p_{1}}=0 & \text { при } & p_{0}+p_{1}+m \neq 3 n .
\end{array}
$$

Представим целое число $p_{0}+p_{1}$ в виде $p_{0}+p_{1}=3 k+r$, где $k$ целое, a $r \in\{-1,0,1\}$. Подставляя (38) в (37), находим

$$
\begin{aligned}
\sum_{\mu=0}^{k}(-1)^{\mu} S_{3 \mu} b_{i}^{-3 \mu}=0 & \text { для } & r=0, \\
\sum_{\mu=0}^{k-1}(-1)^{\mu} S_{3 \mu+2} b_{i}^{-3 \mu}=0 & \text { для } & r=1, \\
\sum_{\mu=0}^{k-1}(-1)^{\mu} S_{3 \mu+1} b_{i}^{-3 \mu}=0 & \text { для } & r=-1,
\end{aligned}
$$


где $i=1,2, \ldots, p_{0}+p_{1}$. Очевидно, что подсистема (29) выполняется, если имеет место равенство

$$
S_{3 \nu+\rho}=0, \quad \nu=0,1, \ldots, k-1,
$$

где $\rho=2$ для $r=1$ и $\rho=1$ для $r=-1$. Поскольку $S_{0}=1$, ограничим рассмотрение случаем $p_{0}+p_{1} \neq 3 k$.

Выберем $p_{1}$ переменных $u_{i}$ так, чтобы выполнялись условия свободных фермионов (30). Теперь переменные $v_{i}, i=1,2, \ldots, p_{0}$, могут быть найдены из системы (40). Используем декомпозицию симметричных функций, зависящих от объединения двух множеств переменных,

$$
\begin{aligned}
& S_{0}=1 \text {, } \\
& S_{1}=s_{1}+\sigma_{1} \text {, } \\
& S_{2}=s_{2}+s_{1} \sigma_{1}+\sigma_{2} \text {, } \\
& S_{m}=\sum_{n=\max \left\{0, m-p_{0}\right\}} s_{m-k} \sigma_{k}, \\
& S_{p_{0}+p_{1}}=s_{p_{0}} \sigma_{p_{1}},
\end{aligned}
$$

где симметричные функции $s_{i}$ зависят от неизвестных переменных $v_{j}$, а значения симметричных функций $\sigma_{i}$ определяются выбранными значениями переменных $u_{j}$. Таким образом, система (40) сводится к линейной системе для симметричных функций $s_{i}, i=$ $1,2, \ldots, p_{0}$. Потребуем, чтобы число переменных $p_{0}$ было больше или равно числу уравнений $k$. Получаем систему условий на целочисленные параметры, фиксирующие сектор, т.е. на числа частиц определенного сорта,

$$
\begin{gathered}
p_{0}=n_{0}, \quad p_{1}=n_{0}+n_{1}, \quad n_{0} \leqslant n_{1}, \\
p_{0}+p_{1}=3 k \pm 1, \quad k \leqslant p_{0} .
\end{gathered}
$$

Система легко сводится к паре уравнений $n_{0}=k, n_{1}=k+1$, и, следовательно, мы оказываемся в секторе $(k, k+1, L-2 k-1)$, который и фигурирует в гипотезе $1 . \mathrm{B}$ соответствующих уравнениях Бете мы имеем $2 k+1$ переменных $u_{i}$ и $k$ переменных $v_{i}$. Значения первой системы переменных (и, тем самым, энергия) выбираются, исходя из условий свободных фермионов (30). Для нахождения второй системы переменных надо использовать линейную систему, состоящую из $k$ уравнений $(40)$ с $\rho=2$. Рассмотрим для иллюстрации случай $k=2\left(p_{0}=2\right.$ и $\left.p_{1}=5\right)$. Имеем пять переменных $u_{1}, \ldots, u_{5}$, которые выбираются так, чтобы выполнялись условия (30), и две переменные $v_{1}, v_{2}$. Используя (41), мы можем представить (40) в виде

$$
\begin{aligned}
& S_{2}=s_{2}+s_{1} \sigma_{1}+\sigma_{2}=0, \\
& S_{5}=s_{5}+s_{4} \sigma_{1}+s_{3} \sigma_{2} .
\end{aligned}
$$

Значения $s_{1}, \ldots, s_{5}$ находим по выбранным значениям $u_{1}, \ldots, u_{5}$, а для $\sigma_{1}=v_{1}+v_{2}$ и $\sigma_{2}=v_{1} v_{2}$ имеем линейную систему (43). Далее используем теорему Виета. 


\section{7. РЕШЕНИЯ УРАВНЕНИЙ БЕТЕ ДЛЯ КВАНТОВО-ИНВАРИАНТНОЙ $S U_{q}(3)$-МОДЕЛИ}

Случай свободных граничных условий более сложен. Подставляя определение (17) в подсистему (29) и избавляясь от знаменателей, получаем

$$
\begin{aligned}
& \sin \left(2 x_{i}-\frac{2 \pi}{3}\right) \prod_{j=1}^{p_{0}+p_{1}}\left[\cos \left(2 x_{j}\right)-\cos \left(2 x_{i}-\frac{2 \pi}{3}\right)\right]+ \\
& \quad+\sin \left(2 x_{i}+\frac{2 \pi}{3}\right) \prod_{j=1}^{p_{0}+p_{1}}\left[\cos \left(2 x_{j}\right)-\cos \left(2 x_{i}+\frac{2 \pi}{3}\right)\right]=0, \quad i=1,2, \ldots, p_{0}+p_{1} .
\end{aligned}
$$

Вновь используя симметричные функции

$$
\begin{aligned}
S_{0} & =1, \\
S_{1} & =\cos 2 x_{1}+\cos 2 x_{2}+\cos 2 x_{p_{0}+p_{1}}, \\
\ldots \ldots \ldots \ldots \ldots \ldots \ldots \ldots \ldots \ldots \ldots \ldots \ldots \ldots \ldots \ldots \ldots \ldots \ldots \ldots \ldots & \ldots \ldots \ldots+\cos 2 x_{p_{0}+p_{1}},
\end{aligned}
$$

можно переписать уравнение (44) в виде

$$
\begin{aligned}
\sum_{m=0}^{p_{0}+p_{1}} S_{p_{0}+p_{1}-m}\left[\sin \left(2 x_{i}-\frac{2 \pi}{3}\right) \cos ^{m}\left(2 x_{i}-\frac{2 \pi}{3}\right)+\right. \\
\left.\quad+\sin \left(2 x_{i}+\frac{2 \pi}{3}\right) \cos ^{m}\left(2 x_{i}+\frac{2 \pi}{3}\right)\right]=0, \quad i=1,2, \ldots, p_{0}+p_{1} .
\end{aligned}
$$

Кроме того, выполняется равенство

$$
\begin{gathered}
\sin 2 x_{i} \prod_{j=1}^{p_{0}+p_{1}}\left(\cos 2 x_{j}-\cos 2 x_{i}\right) \equiv \sum_{m=0}^{p_{0}+p_{1}} S_{p_{0}+p_{1}-m} \sin 2 x_{i} \cos ^{m} 2 x_{i}=0 \\
i=1,2, \ldots, p_{0}+p_{1}
\end{gathered}
$$

Прибавим его к уравнению

$$
\sum_{m=0}^{p_{0}+p_{1}} S_{p_{0}+p_{1}-m} \phi_{m}\left(x_{i}\right)=0, \quad i=1,2, \ldots, p_{0}+p_{1},
$$

где функции $\phi_{m}(x)$ определяются формулой

$$
\phi_{m}(x)=\sum_{\rho=-1}^{1} \sin \left(2 x+\frac{2 \pi \rho}{3}\right) \cos ^{m}\left(2 x+\frac{2 \pi \rho}{3}\right) .
$$


Удобно использовать рекуррентное соотношение, которому удовлетворяют функции $\phi_{m}(x)$. Рассмотрим последовательность

$$
y_{m}=a \alpha^{m}+b \beta^{m}+c \gamma^{m} .
$$

Хорошо известно, что она удовлетворяет рекуррентному соотношению

$$
y_{m+1}+A y_{m}+B y_{m-1}+C y_{m-2}=0,
$$

где функция

$$
t^{3}+A t^{2}+B t+C \equiv(t-\alpha)(t-\beta)(t-\gamma)
$$

является полиномом с корнями $\alpha, \beta, \gamma$. Отсюда следует, что функции $\phi_{m}(x)$ удовлетворяют рекурсии

$$
\phi_{m+1}(x)+A \phi_{m}(x)+B \phi_{m-1}(x)+C \phi_{m-2}(x)=0,
$$

где

$$
t^{3}+A t^{2}+B t+C \equiv \prod_{\rho=-1}^{1}\left[t-\cos \left(2 x+\frac{2 \pi \rho}{3}\right)\right]=t^{3}-\frac{3}{4} t-\frac{1}{4} \cos 6 x .
$$

Согласно формуле (51) имеем следуюшую рекурсию для $\phi_{m}$ :

$$
\phi_{m+1}(x)=\frac{3}{4} \phi_{m-1}(x)+\frac{1}{4} \cos 6 x \phi_{m-2}(x)=0 .
$$

Нам нужны также начальные данные $\phi_{0}, \phi_{1}$ и $\phi_{2}$. Используя определение (49), мы легко получаем эти функции:

$$
\phi_{0}(x)=0, \quad \phi_{1}(x)=0, \quad \phi_{2}(x)=\frac{3}{4} \sin 6 x .
$$

Приведем, для наглядности, список нескольких первых функций $\phi_{i}(x)$, который мы получили, используя начальные данные (56) и рекурсию (55),

$$
\begin{gathered}
\phi_{3}(x)=0, \quad \phi_{4}(x)=\frac{9}{16} \sin 6 x, \quad \phi_{5}(x)=\frac{3}{16} \sin 6 x \cos 6 x, \\
\phi_{6}(x)=\frac{27}{64} \sin 6 x, \quad \phi_{7}(x)=\frac{9}{32} \sin 6 x \cos 6 x, \\
\phi_{8}(x)=\sin 6 x\left(\frac{81}{256}+\frac{3}{64} \cos ^{2} 6 x\right) .
\end{gathered}
$$

Ясно, что $\phi_{m}(x) \equiv \sin 6 x \Phi_{m}(\cos 6 x)$, где $\Phi_{m}(t)$ является многочленом. Степень этого многочлена $\mu$ зависит от его номера $m$. Можно показать по индукции, что если $m=3 n+$ $r, r=1,2,3$, то $\mu=n-2+r$ для $n-2+r \geqslant 0$. Если же $n-2+r<0$, то $\phi_{m}(x) \equiv 0$.

Вернемся к уравнению (48). Используя формулу для $\mu$, мыполучаем, что левая часть этого уравнения является (с точностью до общего множителя $\sin 6 x$ ) полиномом от 
$\cos 6 x$, имеюшим степень $\nu=\left[\left(p_{0}+p_{1}-2\right) / 3\right]$, где $[x]$ означает целую часть числа $x$. Рассмотрим вспомогательное уравнение

$$
\sum_{m=0}^{p_{0}+p_{1}} S_{p_{0}+p_{1}-m} \phi_{m}(x)=0 .
$$

Если мы подберем симметричные комбинации $S_{i}$ так, что последнее уравнение превратится в тождество по переменной $x$, то тем самым удовлетворим и системе (48). Для этого приравняем нулю все коэффициенты полинома

$$
\sum_{m=0}^{p_{0}+p_{1}} S_{p_{0}+p_{1}-m} \Phi_{m}(t)=0
$$

В результате получим $k=\nu+1=\left[\left(p_{0}+p_{1}+1\right) / 3\right]$ линейных уравнений для симметричных комбинаций $S_{m}$. Рассмотрим сначала набор частных случаев, $p_{0}+p_{1}=6,7,8$. Подставляя формулы из списка (57) в уравнение (59), получаем

$$
\begin{gathered}
S_{4}+\frac{3}{4} S_{2}+\frac{1}{4} S_{1} \cos 6 x+\frac{9}{16}=0, \quad p_{0}+p_{1}=6 \\
S_{5}+\frac{3}{4} S_{3}+\frac{1}{4} S_{2} \cos 6 x+\frac{9}{16} S_{1}+\frac{3}{8} \cos 6 x=0, \quad p_{0}+p_{1}=7 \\
S_{6}+\frac{3}{4} S_{4}+\frac{1}{4} S_{3} \cos 6 x+\frac{9}{16} S_{2}+\frac{3}{8} S_{1} \cos 6 x+\frac{27}{64}+\frac{1}{16} \cos ^{2} 6 x=0, \quad p_{0}+p_{1}=8
\end{gathered}
$$

Для $p_{0}+p_{1}=6$ и $p_{0}+p_{1}=7$ мы получаем по два уравнения:

и

$$
S_{1}=0, \quad S_{4}+\frac{3}{4} S_{2}+\frac{9}{16}=0
$$

$$
S_{2}+\frac{3}{4}=0, \quad S_{5}+\frac{3}{4} S_{3}+\frac{9}{16} S_{1}=0,
$$

соответственно. Что касается случая $p_{0}+p_{1}=8$, то последний член $1 / 16 \cos ^{2} 6 x$ не позволяет превратить равенство (59) в тождество.

Обратимся теперь к обшему случаю. Как и в случае с периодическими граничными условиями, мы фиксируем переменные $u_{i}, i=1, \ldots, p_{1}$, так, чтобы они удовлетворяли условию свободных фермионов (30), а потом пытаемся найти переменные $v_{i}, i=$ $1, \ldots, p_{0}$, используя $k=\left(p_{0}+p_{1}+1\right) / 3$ уравнений, которые мы получим, приравнивая нулю все коэффициенты полинома (59). Число уравнений $k$ не должно превышать число переменных $p_{0}$. Учитывая это условие и все ограничения на параметры, фиксируюшие сектор, получаем систему соотношений

$$
p_{0}=n_{0}, \quad p_{1}=n_{0}+n_{1}, \quad n_{0} \leqslant n_{1}, \quad p_{0} \geqslant \frac{1}{3}\left(p_{0}+p_{1}+1\right) .
$$

Эта система имеет два возможных решения:

a) $n_{0}=n_{1}=k, p_{0}+p_{1}=3 k$, что соответствует секторам $(k, k, L-2 k)$;

б) $n_{0}=k, n_{1}=k+1, p_{0}+p_{1}=3 k+1$, что соответствует секторам $(k, k+1, L-2 k-1)$. Используя рекуррентные соотношения (55) совместно с начальными данными (56), можно показать, что при $p_{0}+p_{1} \neq 3 k+2$ мы всегда получаем линейную систему для симметричных комбинаций $S_{i}$, число уравнений которой равно числу неизвестных. Находя эти комбинации, мы можем использовать их так же, как мы использовали систему (40). 


\section{8. ОБЩИЙ МЕТОД ПОСТРОЕНИЯ СВОБОДНО-ФЕРМИОННЫХ СОСТОЯНИЙ}

Ограничимся здесь самыми обшими замечаниями, отсылая интересуюшегося читателя к третьей работе из серии [9].

Мы нашли много свободно-фермионных состояний для $S U_{q}(N)$-моделей с $q=-e^{i \pi / N}$, но лишш одно состояние для $S U_{q}(N-1)$-модели с тем же самым значением $q$ и с энергией (13). Как отмечалось выше, это состояние можно рассматривать как специальный случай $S U_{q}(N)$-модели (см. гипотезу 6). Мы намерены привести аргументы в пользу того, что соответствуюшая волновая функция, во-первых, обладает замечательными комбинаторными свойствами и, во-вторых, играет важную роль при построении решений с помощью "матрешечных" ( “вложенных") уравнений Бете. При этом удается объяснить существование всех свободно-фермионных состояний. Дело в том, что, используя метод вложенных уравнений Бете для $S U_{q}(N)$-инвариантного гамильтониана (1), мы получаем (см., например, [14]) вспомогательную трансфер-матрицу для неоднородной $S U_{q}(N-1)$-инвариантной вершинной модели. Мы предполагаем, что эта матрица имеет уникальное факторизуемое собственное значение. Именно это собственное значение и приводит часть уравнений Бете к простому свободно-фермионному виду. Как следствие мы получаем, что часть спектра гамильтониана (1) носит свободно-ффермионный характер и что все соответствуюшие собственные векторы могут быть выражены через одну волновую функцию, соответствующую вышеупомянутому уникальному факторизуемому собственному значению вспомогательной трансфер-матрицы для неоднородной $S U_{q}(N-1)$. Для однородного случая трансфер-матрица коммутирует с $S U_{q}(N-$ 1)-инвариантным гамильтонианом и волновая функция соответствует вышеупомянутому основному состоянию с энергией (13).

Что касается случая с периодическими граничными условиями, то мы обнаружили свободно-фермионный спектр лишь для $S U_{q}(3)$-модели Перка-Шульц с параметром $q=e^{2 i \pi / 3}$ (мы не рассматриваем здесь знаменитую $X Y$-спиновую модель). В разделе 4 заключительной работы из серии [9] было показано, что этот спектр легко объясняется существованием уникального собственного значения для трансфер-матрицы неоднородной 6 -вершинной модели с этим же значением $q$ (см. также гипотезу 3 ). В свою очередь, сушествование этого уникального собственного значения удалось доказать, используя различные методы, описанные в работах [9], [15].

Вернемся к обсуждению квантово-инвариантного случая. Известно, что собственное значение первой вспомогательной трансфер-матрицы, соответствующей неоднородной $S U_{q}(N-1)$-инвариантной модели на квадратной решетке ширины $p$, удовлетворяет формуле (см. уравнение (51) из работы [14]) ${ }^{2}$

$$
\Lambda_{\mathrm{aux}}^{(N-1)}\left(u_{i}\right)=f^{-L}\left(u_{i}, 0\right) \prod_{j=1, j \neq i}^{p} F\left(u_{i}, u_{j}\right), \quad i=1, \ldots, p
$$

\footnotetext{
2) Все детали, относящиеся к конструкции трансфер-матрицы, могут быть найдены в работе [14].
} 
Мы видим, что условия свободных фермионов (19) являются следствием сушествования специального собственного значения этой матрищы

$$
\Lambda_{\operatorname{aux}}^{(N-1)}(u)=F^{-1}(u, u) \prod_{j=1}^{p} F\left(u, u_{j}\right) .
$$

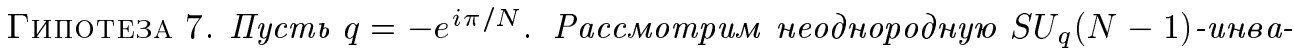
риантную вершинную модель на квадратной решетке с р вертикальными линиями, где $p=(N-1) k+r, \quad 0 \leqslant r \leqslant N-2$. Неоднородности модели фиксируются вертикальными бистротами $u_{j}, j=1, \ldots, p$. Тогда горизонтальная трансфер-матрица, зависящая от спектрального параметра и (от горизонтальной бистроты), имеет специальное факторизуемое собственное значение, даваемое формулой (67). Соответствующий собственный вектор принадлежит сектору $S=\left(n_{0}, n_{1}, \ldots, n_{N-2}\right)$, әде $n_{i}=k, i=0, \ldots, r-1, u n_{i}=k+1$ для $i=r, \ldots, N-2$.

\section{9. КОМБИНАТОРНЫЕ СВОЙСТВА ВОЛНОВОЙ ФУНКЦИИ ОСНОВНОГО СОСТОЯНИЯ $S U_{q}(N-1)$-ИНВАРИАНТНОЙ МОДЕЛИ ПРИ $q=-e^{i \pi / N}$.}

Представляется, что гипотеза 7 наиболее естественным образом объясняет появление свободно-фермионного спектра именно в специальных секторах (см. гипотезы 4 и $4^{\prime}$ ). Хотя мы не имеем доказательства этой гипотезы, мы можем привести некоторые аргументы в ее пользу. Как говорилось в предыдущем разделе, в заключительной работе из серии [9] был доказан аналог гипотезы 7 для $S U_{q}(2)$-модели с периодическими граничными условиями.

УТВЕРЖДЕНИЕ. Пусть $q=e^{2 i \pi / 3}$. Рассмотрим неоднородную 6-вериинную модель на квадратной решетке с $2 k+1$ вертикальными линиями. Неоднородности модели фиксируются вертикальными быстротами $u_{j}, \quad j=1, \ldots, 2 k+1$. Тогда горизонтальная трансфер-матрица, зависящая от спектрального параметра $и$ (от горизонтальной быстроты), имеет специальное факторизуемое собственное значение. Соответствующий собственный вектор принадлежст сектору $S=(k, k+1)$.

Было найдено [7], что компоненты этого собственного вектора обладают замечательными комбинаторными свойствами. По аналогии мы решили исследовать комбинаторные свойства волновых функций из гипотезы 7. Для простоты мы ограничились однородным случаем и нашли, в частности, что отношение, определяемое формулой

$$
R=\frac{\left(\sum_{i} v_{i}\right)^{2}}{\sum_{i} v_{i}^{2}}
$$

где $v_{i}$ - компоненты волновой функции, по всей вероятности, принимает простые значения. 
Для $X X Z$-цепочки длины $L$, которая непосредственно связана с однородной 6-вершинной моделью, было найдено, что при $q=e^{2 i \pi / 3}$ имеется простое значение $R=(\sqrt{3})^{\alpha}$, где $\alpha=L-1$ для нечетных значений $L$ и периодической границы или для $S U_{q}(2)$-инвариантного случая. С другой стороны, $\alpha=L$ для четных значений $L$. При этом мы должны рассматривать скрученные граничные условия или $S U_{q}(2)$-инвариантный случай. Представим эти результаты в компактной форме

$$
\frac{R_{L+2}}{R_{L}}=3, \quad R_{1}=1, \quad R_{2}=3 .
$$

Используя высокоточную численную диагонализацию с последуюшим фитированием специальными иррациональными числами, мы получили значения $R$ в следующих случаях:

a) $S U(3), q=-e^{i \pi / 4}, 2 \leqslant L \leqslant 9$,

$$
\begin{gathered}
\frac{R_{L+3}}{R_{L}}=(1+\sqrt{2})^{3}, \\
R_{1}=1, \quad R_{2}=1+\sqrt{2}, \quad R_{3}=(1+\sqrt{2})^{3} ;
\end{gathered}
$$

б) $S U(4), q=-e^{i \pi / 5}, 2 \leqslant L \leqslant 8$,

$$
\begin{gathered}
\frac{R_{L+4}}{R_{L}}=(5+2 \sqrt{5})^{2}, \\
R_{1}=1, \quad R_{2}=\sqrt{5}, \quad R_{3}=5+2 \sqrt{5}, \quad R_{4}=(5+2 \sqrt{5})^{2} ;
\end{gathered}
$$

в) $S U(5), q=-e^{i \pi / 6}, 2 \leqslant L \leqslant 7$,

$$
\begin{gathered}
\frac{R_{L+5}}{R_{L}}=(2+\sqrt{3})^{5}, \\
R_{1}=1, \quad R_{2}=\frac{2+\sqrt{3}}{\sqrt{3}}, \quad R_{3}=\frac{(2+\sqrt{3})^{2}}{\sqrt{3}}, \\
R_{4}=(2+\sqrt{3})^{3}, \quad R_{5}=(2+\sqrt{3})^{5} .
\end{gathered}
$$

Значения $R_{L}, L<N$, которые необходимы для развертывания этих рекуррентных соотношений, могут быть найдены теоретически по точным значениям соответствующих волновых функций (см. приложение В второй работы серии [9]). Можно показать, что для $L<N$

$$
R_{L}=\left(\frac{1+x}{1-x}\right)^{L-1} \prod_{k=2}^{L} \frac{1-x^{k}}{1+x^{k}}, \quad x=-q=e^{i \pi / N} .
$$

Что касается случаев $L \geqslant N$, то мы не нашли отклонения от замечательного соотношения

$$
R_{L+N-1}=R_{N-1} R_{L}
$$

которое, как и большая часть наших результатов, ждет доказательства или опровержения.

Благодарности. Автор благодарен оргкомитету Семинара за приглашение. Работа поддержана грантами РФФИ № 01-01-00201 и INTAS № 00-00561. 


\section{Список литературы}

[1] H. A. Bethe. Z. Phys. 1931. V. 71. P. 205-226.

[2] Р. Бакстер. Точно решаемые модели в статистической механике. М.: Мир, 1986; V. E. Korepin, I. G. Izergin, N. M. Bogoliubov. Quantum Inverse Scattering Method, Correlation Functions and Algebraic Bethe Ansatz. Cambridge: Cambridge Univ. Press, 1993; F. H. L. Essler, V. E. Korepin. Exactly Solvable Models of Strongly Correlated Electrons. Singapore: World Scientific, 1994; P. Schlottmann. Int. J. Mod. Phys. B. 1997. V. 11. P. 355-667.

[3] F. C. Alcaraz, M. N. Barber, M. T. Batchelor. Ann. Phys. 1988. V. 182. P. 280-343; F. C. Alcaraz, M.N. Barber, M. T. Batchelor, R.J. Baxter, G.R.W. Quispel. J. Phys. A. 1987. V. 20. P. 6397-6409.

[4] R. J. Baxter. Adv. Stud. Pure. Math. 1989. V. 19. P. 95-116.

[5] V. Fridkin, Yu. G. Stroganov, D. Zagier. J. Phys. A. 2000. V. 33. P. L121-L125; J. Stat. Phys. 2001. V. 102. P. 781-794; nlin.SI/0010021; Yu. G. Stroganov. J. Phys. A. 2001. V. 34. P. L179-L186; cond-mat/0012035.

[6] R. J. Baxter. Stud. Appl. Math. 1971. V. 50. P. 51-69.

[7] A. V. Razumov, Yu. G. Stroganov. J. Phys. A. 2001. V. 34. P. 3185-3190; cond-mat/0012141; M. T. Batchelor, J. de Gier, B. Nienhuis. J. Phys. A. 2001. V. 34. P. L265-L270; cond-mat/0101385; A. V. Razumov, Yu. G. Stroganov. J. Phys. A. 2001. V. 34. P. 5335-5340; cond-mat/0102247; Combinatorial nature of ground state vector of $O(1)$ loop model. math.CO/0104216; P. A. Pearce, V. Rittenberg, J. de Gier. Critical $Q=1$ Potts model and Temperly-Lieb stochastic processes. cond-mat/0108051; A. V. Razumov, Yu. G. Stroganov. $\mathrm{O}(1)$ loop model with different boundary conditions and symmetry classes of alternating-sign matrices. cond-mat/0108103; J. de Gier, M. T. Batchelor, B. Nienhuis, S. Mitra. The $X X Z$ spin chain at $\Delta=-1 / 2$ : Bethe roots, symmetric functions and determinants. math-ph/0110011; Ю.Г. Строганов. ТМФ. 2001. Т. 129. № 2. С. 345-359; N. Kitanine, J. M. Maillet, N. A. Slavnov, V. Terras. J. Phys. A. 2002. V. 35. P. L385-L391; hep-th/0201134; M. T. Batchelor, J. de Gier, B. Nienhuis. Int. J. Mod. Phys. B. 2002. V. 16. P. 1883-1890; math-ph/0204002; J. de Gier, B. Nienhuis, P. A. Pearce, V. Rittenberg. Phys. Rev. E. 2003. V. 67. P. 016101-016104; cond-mat/0205467.

[8] Yu.G. Stroganov. The 8-vertex model with a special value of the crossing parameter and the related $X Y Z$ spin chain. In: Integrable Structures of Exactly Solvable Two-Dimensional Models of Quantum Field Theory. Eds. S. Pakuliak, G. von Gehlen. Amsterdam: Kluwer, 2001. P. 315-319.

[9] F. C. Alcaraz, Yu. G. Stroganov. J. Phys. A. 2002. V. 35. P. 3805-3820; cond-mat/0201354; 2002. V. 35. P. 6767-6788; cond-mat/0205567; 2003. V. 36. P. 2381-2397; cond-mat/0212475.

[10] J. H. H. Perk, C. L. Schultz. Phys. Lett. A. 1981. V. 84. P. 407-410; C. L. Schultz. Physica A. 1983. V. 122. P. 71-88.

[11] B. Sutherland. Phys. Rev. B. 1975. V. 12. P. 3795-3805.

[12] N. Y. Reshetikhin, P. B. Wiegmann. Phys. Lett. B. 1987. V. 189. P. 125-131; H. J. de Vega. Int. J. Mod. Phys. A. 1989. V. 4. P. 2371-2463.

[13] L. Mezincescu, R.I. Nepomechie, P.K. Towsend, A. M. Tsvelick. Nucl. Phys. B. 1993. V. 406. P. 681-707.

[14] H. J. de Vega, A. Gonzáles-Ruiz. Nucl. Phys. B. 1994. V. 417. P. 553-578.

[15] Yu. G. Stroganov. A new way to deal with Izergin-Korepin determinant at root of unity. math-ph/0204042. 